\title{
Evidências de Validade de uma Medida de Liderança Abusiva
}

\author{
Katia Puente-Palacios ${ }^{1}$ \\ Universidade de Brasília, Brasília-DF, Brasil \\ Marcela Guimarães Côrtes \\ Instituto Nacional de Estudos e Pesquisas Educacionais Anísio Teixeira (INEP), Brasília-DF, Brasil
}

\section{RESUMO}

A ocorrência de liderança abusiva constitui fato de enorme saliência, pois se trata de atuação oposta à esperada de um líder. Envolve um conjunto de comportamentos desviantes percebidos pelo subordinado, dos quais ele é o alvo. O estudo dessa forma de liderança se justifica pelas consequências nocivas que desencadeia. Todavia, a sua identificação depende da disponibilidade de uma medida confiável. Assim, o objetivo do presente estudo é apresentar as evidências de validade de uma medida de liderança abusiva. A amostra da pesquisa contou com 363 participantes que também forneceram informações sobre satisfação e intenções de rotatividade. Os resultados obtidos revelam boas propriedades psicométricas da escala, com solução unifatorial (56,79 de \% variância explicada; $\alpha=0,94)$. Como esperado, a liderança abusiva mostrou-se negativamente associada com a satisfação e positivamente associada com a intenção de rotatividade. Esses resultados evidenciam a pertinência da medida como ferramenta de diagnóstico da liderança abusiva.

Palavras chave: liderança abusiva; análise fatorial; mensuração.

\section{ABSTRACT - Validity Evidences of a Measure of Abusive Leadership}

The occurrence of abusive leadership is a fact of great prominence, since it concerns behavior which is the opposite of what is expected of a leader. It involves several deviant behaviors perceived by subordinates as targeting them. Studying this type of abusive leadership behavior is justified due to its consequences. However, this depends on the availability of a reliable measure. In this context the aim of this study is to present validity evidence for a measure of abusive leadership. The study sample consisted of 363 participants that provided information on jobs satisfaction and turnover intention. The results obtained provided satisfactory evidence for the psychometric properties of the scale, with a single factor solution (56.8\% explained variance, $\alpha=.94)$. As expected, abusive leadership showed a negative correlation with job satisfaction and a positive correlation with turnover intention. These results highlight the relevance of the measure as a diagnostic tool for abusive leadership.

Keywords: abusive leadership; factor analysis; measurement.

\section{RESUMEN - Evidencias de Validad de una Medida de Liderazgo Abusivo}

La ocurrencia de liderazgo abusivo constituye un hecho de significativa importancia, dado que se trata de actuación opuesta a lo esperado de un líder. Implica un conjunto de comportamientos desviados percibidos por el subordinado, de los cuales él es el objetivo. El estudio de dicho tipo de liderazgo se justifica por las consecuencias nocivas que desencadena. Sin embargo, su identificación depende de la disponibilidad de una escala fiable. Por consiguiente, el objetivo del presente estudio es presentar evidencias de validez de una escala de liderazgo abusivo. La muestra del estudio contó con 363 participantes que ofrecieron informaciones sobre satisfacción e intenciones de rotación. Los resultados demostraron propiedades psicométricas adecuadas de la escala, con solución unifactorial $(56,79$ de $\%$ varianza explicada; $\alpha=0,94)$. Como esperado, el liderazgo abusivo se manifestó negativamente asociado con la satisfacción y positivamente relacionado con la intención de rotación. Estos resultados evidencian la adecuación de la escala como herramienta de diagnóstico del liderazgo abusivo.

Palabras clave: liderazgo abusivo; análisis factorial; mensuración.

O estudo da supervisão abusiva e as suas consequências têm surgido nas publicações do campo da psicologia e da administração, com maior frequência, nos últimos quinze anos. A ocorrência desse abuso, de difícil diagnóstico tendo em vista a sensibilidade do assunto focado, acarreta consequências severas, tanto para o funcionário vítima do supervisor, como para a organização em que acontece. Assim, as consequências danosas que a sua ocorrência desencadeia são diversas e realçam a importância de investir no seu estudo. A conceituação ou 
descrição do que seja a supervisão abusiva foi realizada inicialmente por Tepper (2000), quem destaca se tratar da "percepção do subordinado relativa ao engajamento do supervisor em comportamentos hostis dirigidos a ele, sendo esses de natureza verbal e não verbal, excluindo ações de contato físico" (p. 178). Ainda que eventos de natureza similar possam ser identificados no cenário organizacional, como liderança antiética, supervisão tirânica ou ainda bullying organizacional, a supervisão abusiva tem mostrado, enquanto construto teórico, um claro conjunto de atributos que permitem afirmar que se trata de fenômeno efetivamente diferenciado desses. Portanto, o seu estudo, diagnóstico e monitoramento constituem ações relevantes e necessárias na busca de melhor compreensão dos antecedentes que levam à sua ocorrência. Ainda assim, condição necessária para esse entendimento é a existência de uma medida confiável que permita mapear a sua presença no cenário organizacional. Desse modo, o objetivo deste estudo é demonstrar as evidências de validade da escala de avaliação de supervisão abusiva proposta por Tepper, tanto em termos dos atributos do construto, como da sua vinculação com outros fenômenos, de acordo com as bases teóricas em que se sustenta, sendo evidenciada, desse modo, a validade concorrente.

A importância do estudo dessa forma de supervisão é defendida tanto pela natureza do comportamento do ator que comete o abuso, qualificado como sendo, no mínimo, inapropriado, mas especialmente pelas diversas consequências nocivas que a sua ocorrência acarreta. Entretanto, a compreensão dos efeitos nocivos precisa ser antecedida do claro estabelecimento das características do construto, pois é necessário definir os limites conceituais que o diferenciam de outros fenômenos que coexistem no cenário organizacional.

Ainda, antes de adentrar na discussão do que seja a supervisão abusiva, é importante destacar que os termos "liderança" e "supervisão" podem ser encontrados como descritores do mesmo fenômeno. Contudo, o autor que originalmente propôs uma mediada de avaliação da sua ocorrência (Tepper, 2000) faz referência à supervisão abusiva (abusive supervision, em inglês), enquanto a literatura científica publicada em língua portuguesa tende a adotar a palavra liderança (Garrido, 2012). Desse modo, neste artigo, ambos os termos serão usados, sem desconhecer o papel diferenciado do supervisor e do líder, nas organizações, uma vez que essa discussão foge do escopo deste trabalho.

Quanto às características da supervisão abusiva, essa pode ser descrita pela presença de um conjunto de comportamentos percebidos pelo subordinado. Assim, a rigor, o foco de interesse não é o ator que comete o abuso, mas aquele que o percebe (Pradhan \& Jena, 2016). Portanto, um dos atributos desse fenômeno é o fato de ser uma vivência de natureza subjetiva do trabalhador, que considera ter sido alvo de abuso por parte do seu líder (Tepper, 2007).
Além de ser descrita como uma vivência subjetiva, a liderança abusiva tem sido caracterizada por fazer referência à percepção de continuidade dos comportamentos hostis do supervisor. Portanto, não se trata de um episódio isolado de maltrato ao trabalhador (Pradhan \& Jena, 2016), mas assume o caráter de abuso continuado. Um terceiro aspecto é relativo ao fato de o comportamento abusivo do líder não ser isento de um objetivo (Tepper, 2000). Ainda assim, o objetivo perseguido não é relacionado ao trabalhador, mas ao trabalho. Desse modo, o que o supervisor busca é viabilizar a realização da tarefa ou mostrar um padrão de desempenho considerado inadequado (Tepper, 2007), e não necessariamente cometer abuso contra alguém especificamente. Assim, o abuso é descrito como tendo um caráter instrumental, embora estudos mais recentes façam questionamentos a esse aspecto (Walter, van der Vegt, Huang, \& Miao, 2015). Por fim, a ausência de contato físico é uma característica relevante do construto em tela (Martinko, Sikora, \& Harvey, 2012). A supervisão abusiva, por definição, não envolve agressão física, de tal sorte que a sua manifestação ocorre mediante a adoção de padrões verbais ou gestuais. Esse conjunto de características do abuso (subjetivo, continuado, com objetivo definido e sem contato físico) ajuda a estabelecer os limites teóricos do construto, os quais devem ser observados em uma medida que busca propiciar o seu diagnóstico.

Conforme apontado, o abuso se manifesta pela adoção de padrões comportamentais por parte do líder, entre os que têm sido descritos, por exemplo, trato rude ou desrespeitoso, fazer críticas na frente de outras pessoas, ridicularizar o funcionário pela falta de competências ou habilidades, e constranger ou humilhar o empregado em público (Bies, 2000). Todavia, seguindo a lógica teórica da liderança abusiva, pressupõe-se não somente a ocorrência desses comportamentos, mas também a avaliação deles como abuso, realizada pelo trabalhador que se considera o alvo.

Ao explicar a ocorrência dessa percepção e as consequências derivadas, Mackey, Frieder, Brees e Martinko (2017) apontam que a percepção de justiça constitui um elemento de central importância. Desse modo, na proposição original do construto feita por Tepper (2000), a justiça aparece tanto na forma de base teórica explicativa da ocorrência da percepção e abuso sofrido (por parte do subordinado), como também aparece desempenhando o papel de variável moderadora das consequências derivadas da supervisão abusiva sobre diversos resultados, sejam esses do nível individual ou organizacional. Ainda assim, a revisão de estudos empíricos da área revela que diversas outras proposições teóricas são apresentadas como bases explicativas da ocorrência da supervisão abusiva e os seus efeitos. Por exemplo, pesquisadores têm adotado a teoria da troca social (Kim, Kim \& Yun, 2015), a teoria da motivação intrínseca (Zhang, Kwan, Zhang \& Wu, 2014) 
ou ainda a teoria da aprendizagem social (Hon \& Lu, 2016) para explicar a ocorrência de certos consequentes, enquanto teorias como a da vitimização (Walter et al., 2015) ou a dominância social (Khan, Moss, Quratulain \& Hameed, 2016) surgem como propostas explicativas da ocorrência (antecedentes) da supervisão abusiva.

Em pesquisa do tipo meta-análise, realizada buscando identificar os efeitos da supervisão abusiva, os autores (Mackey, Frieder, Brees \& Martinko, 2017) acertadamente alertam que a falta de uma base teórica unificadora dos estudos constitui uma fragilidade do campo, e destacam a saliência da justiça como arcabouço teórico que aparece de maneira mais recorrente para explicar tanto a percepção do comportamento abusivo como para analisar as consequências da sua manifestação. A justiça ora mencionada refere-se à percepção geral do trabalhador sobre o fato dele se sentir valorizado pela organização. Dentre os diversos tipos de justiça propostos (distributiva, procedimental e interacional - Bies, 2000), a justiça interacional aparece como elemento chave na avaliação feita pelo subordinado a respeito da vivência do abuso sofrido por parte do supervisor (Tepper, 2000).

A justiça interacional é apresentada como um tipo diferenciado de justiça, cujo foco recai nas interações mantidas pelo indivíduo no seu local de trabalho, na aplicação dos procedimentos organizacionais. Pontualmente, é defendido que as "pessoas se preocupam com o tratamento interpessoal que recebem dos outros" (...) e "essas preocupações são distintas das relativas à justiça distributiva e procedimental" (Bies, 2000, p. 63). No caso da supervisão abusiva, a percepção do trabalhador seria a de que o tratamento recebido por parte do líder viola princípios de respeito, honestidade, adequação e cuidado com os sentimentos pessoais (Tepper, 2000). Esses princípios seriam esperados por caracterizarem um tratamento justo (justiça interacional).

Por outro lado, é pontuado que comportamentos descritos como rudes podem ser considerados aceitáveis em cenários ou situações específicas ou como parte de determinados processos; por exemplo, treinamentos militares ou intimidação a candidatos a ocupar cargos cujas tarefas são caracterizadas por elevada pressão. Assim, na avaliação do comportamento do supervisor como sendo uma demonstração de injustiça, o julgamento do trabalhador é o aspecto central.

A justiça interacional, portanto, oferece o suporte teórico que explica as expectativas que o sujeito constrói sobre o tratamento que deve ser dado pelo líder. Quando os princípios de justiça esperados pelo trabalhador são violados, e não são vislumbradas justificativas plausíveis para a sua ocorrência, o resultado seria a percepção de ocorrência de abuso.

Além de descrever as propriedades teóricas da supervisão abusiva, enquanto construto psicológico, é importante destacar o que faz esse construto diferente de outros comportamentos desviantes vistos no local de trabalho. Um fenômeno que tem cobrado cada vez mais atenção é o bullying ocorrido no cenário de trabalho. É descrito como um conjunto de práticas ou ações repetidas de maltrato sistemático, que envolvem humilhação e ofensas, e desencadeiam estresse na vítima (Einarsen, Hoel, Zapf \& Cooper, 2011). Esse maltrato, se exercido de maneira continuada sobre o trabalhador, causa consequências severas, tanto de natureza social, como psicológica e/ou psicossomática (Borges \& Ferreira, 2015).

Apesar de ter pontos em comum com a liderança abusiva (maltrato recebido, por exemplo), existem algumas diferenças centrais como a intencionalidade do abuso cometido no bullying, pois nesse o sujeito é definido como alvo a ser atingido. Outra diferença está no fato do bullying poder ser dirigido também em relação às chefias, fato que é contrário à liderança abusiva, que é necessariamente exercida por alguém alocado em escalão hierárquico superior. Por fim, uma diferença adicional é a possibilidade de contato (agressão) físico, reconhecida no bullying, mas que explicitamente extrapola o conceito de supervisão abusiva.

Ainda buscando destacar a especificidade teórico-conceitual da supervisão abusiva, Tepper (2007) a distingue de ações generalizadas de abuso cometido por parte de ator organizacional localizado em patamar hierarquicamente superior; o chamado abuso hierárquico generalizado. Discorrendo sobre essas diferenças, o autor destaca o fato de a supervisão abusiva ter como emissor um líder específico, enquanto, no abuso generalizado, o emissor pode ser simplesmente "algum" chefe. Isso porque a investigação da sua ocorrência se dá indagando os trabalhadores se eles já foram vítimas de abuso cometido por algum chefe, e não por uma pessoa em específico. Uma segunda diferença mencionada diz respeito ao fato de poder abarcar componentes de abuso físico envolvendo, inclusive, assédio sexual ou discriminação.

Com relação às medidas utilizadas para mensurar a liderança abusiva, a proposta da escala original foi realizada por Tepper (2000), quem apresentou um instrumento composto por 15 itens construídos com inspiração em uma medida de relacionamentos abusivos. Esses itens estavam associados a uma escala de frequência (da ocorrência do abuso) que vai de um ("Não lembro que meu chefe tenha mostrado esse comportamento comigo") a cinco ("Meu chefe usa esse comportamento frequentemente comigo"). O conteúdo original dos itens (em inglês) traz afirmativas como: "Tells me my thoughts or feelings are stupid". Na pesquisa de verificação de evidências de validade, essa medida foi aplicada junto com outras 14, e a realização de uma análise fatorial confirmatória (AFC) mostrou ajuste adequado do modelo que condensou 15 fatores. Todos os itens da medida de liderança abusiva se congregaram em um fator único e mostraram cargas fatoriais superiores a 0,5 .

Estudos sobre a temática da liderança abusiva, realizados tomando como base as contribuições teóricas de 
Tepper (2000), são oriundos de uma grande diversidade de países. A revisão de autoria de Martinko, Harvey, Brees e Mackey (2013), por exemplo, atesta a existência de amostras originarias da Austrália, Coreia do Sul, Filipinas, Canadá, só para citar alguns dos referenciados na publicação. Nessa revisão, entretanto, não foram identificados estudos oriundos da América Latina.

Focando pontualmente no comportamento da medida, em estudos que utilizaram a escala de Tepper (2000), constata-se a presença de bons índices psicométricos. Por exemplo, Zhaoquan, Kwan, Qiu, Liu e Yim (2012) aplicaram a escala em uma amostra de trabalhadores chineses e encontraram uma solução unifatorial, com índice satisfatório de confiabilidade $(\alpha=0,93)$. Da mesma forma, Hon e Lu (2016) utilizaram a medida em um estudo realizado com empresas chinesas e também verificaram que os itens se congregaram em fator único $(\alpha=0,92)$. Estudo realizado nos Estados Unidos (Burton, Hoobler \& Scheuer, 2012) com uma amostra de estudantes de um curso de MBA que estava trabalhando, à época da coleta de dados, também identificou estrutura unifatorial da medida $(\alpha=0,91)$. Em outra área geográfica, Moreno, Herrera e Gargurevich (2019) aplicaram a escala a uma amostra de trabalhadores equatorianos e identificaram, do mesmo modo, uma estrutura unifatorial $(\alpha=0,95)$. No Brasil, contudo, não foi encontrada publicação científica relatando o uso da medida. Assim, tanto não há resultados a relatar, como também se constata a existência de uma lacuna que este estudo busca preencher.

Além de mostrar o interesse que o estudo da liderança abusiva tem despertado, assim como destacar o fato da medida original ser usada em diversas pesquisas, cabe também discutir as consequências da liderança. Focando nos consequentes do abuso cometido por líderes, observa-se que as pesquisas empíricas têm destacado tanto os consequentes localizados no nível individual, como no organizacional. Quanto ao nível macro (ou organizacional), dados reportados por Tepper (2007), apontam um custo de aproximadamente 23 bilhões de dólares gastos nos Estados Unidos, por conta de atendimento à saúde e absenteísmo do trabalhador, resultantes de situações de abuso perpetrado pelo supervisor.

No Brasil, lamentavelmente não foram identificados dados específicos quanto às consequências do abuso dos líderes sobre os trabalhadores, mas publicações de pesquisadores nacionais apontam que estresse, tensão e manifestações similares derivam de condições de trabalho, entre as quais pode ser incluído o estilo de supervisão (Borges, Guimarães \& Souza, 2013; Zanelli, 2015). De maneira específica com relação ao estresse vivenciado pelos trabalhadores brasileiros, resultados de levantamento realizado pela International Stress Management Association demonstraram que os trabalhadores brasileiros são os mais estressados, conforme referido pelo site saúde ocupacional ("Brasileiros são os mais estressados," 2016). Assim, pode-se afirmar que, também em solo brasileiro, o abuso dos supervisores, cometido no local de trabalho, tem grande potencial para incorrer em elevado custo financeiro para as organizações e o país, além de prejudicar a saúde e bem-estar dos trabalhadores.

Com relação aos estudos que tomaram como foco o nível individual (os trabalhadores), observa-se uma ampla diversidade de critérios estudados. Assim, metanálise realizada por Martinko et al. (2012) reporta associação significativa positiva entre liderança abusiva e depressão do trabalhador (efeito $=0,21$ ), exaustão emocional $(0,32)$ comportamentos contraproducentes $(0,37)$ e tensão no trabalho $(0,31)$. A importância desses dados está no fato de derivarem de estudo que, pela sua natureza (metanálise), consegue fornecer estimativas menos vulneráveis a associações espúrias ou inflacionadas, resultantes de populações, métodos ou culturas específicas, uma vez que tomam como base conjuntos de amostras de estudos realizados.

A ocorrência da liderança abusiva é vista como prejudicial ao bom funcionamento da organização, não somente pelas consequências que traz sobre os trabalhadores que se percebem como vítimas do abuso, mas também porque a sua ocorrência pode desencadear a imitação de comportamentos abusivos, por parte dos subordinados. Pontualmente, Hon e Lu (2016) argumentam, tomando como base a teoria da aprendizagem social, que o comportamento demostrado pelo líder pode ser visto pelo subordinado como padrão de comportamento aceito pela organização e, portanto, como modelo a ser copiado. Dessa forma, o trabalhador vítima de abuso teria elevada probabilidade de replicar esse comportamento abusivo perante aqueles abaixo da sua linha hierárquica. Os resultados do estudo realizado por esses autores mostraram que o abuso sofrido pelo trabalhador está associado a pior desempenho. Todavia, revelaram também que a imitação do comportamento abusivo pode ser detida em contexto com valores culturais diferentes. Nesse sentido, os autores argumentam que, em culturas caracterizadas por elevada distância de poder, comportamentos rudes (abusivos) podem ser vistos como legítimos do líder, mas não serem vistos como adequados para serem adotados pelo trabalhador e, portanto, não seriam replicados.

Também buscando identificar os consequentes da ocorrência da liderança abusiva, Kernan, Watson, Chen e Kim (2011) investigaram o seu poder preditivo em relação à satisfação dos trabalhadores, envolvimento no trabalho e bem-estar. De maneira similar ao estudo antes relatado, foi previsto que essa relação estaria mediada por variáveis culturais, especificamente relacionados à distância de poder. Novamente a lógica subjacente dessa relação estabelece que, em culturas caracterizadas por elevada distância de poder, comportamentos rudes do supervisor podem ser vistos como atributos do cargo e não como manifestações de abuso. Os resultados obtidos na realização da pesquisa empírica mostraram associação entre abuso, satisfação e bem-estar (efeitos negativos). 
Contudo, na testagem do papel da distância de poder não foram encontrados resultados significativos. Esses achados mostram, portanto, que ainda não está claramente estabelecido o papel de atributos culturais na compreensão do efeito da supervisão abusiva. Ainda assim, as descobertas proporcionadas pelas pesquisas reiteram a pertinência das hipóteses relacionadas ao efeito deletério da supervisão abusiva.

Outro fenômeno que é mencionado nas pesquisas da área como um consequente da liderança abusiva é o turnover (Pradhan \& Jena, 2016). O seu estudo é reiteradamente realizado investigando a intenção de saída da empresa, pois se entende que deixar o emprego nem sempre é uma realidade viável para o empregado, tendo em vista que depende de aspectos como condições do mercado, qualificação do trabalhador e empregabilidade em um cenário específico, só para pontuar alguns. Segundo Pradhan e Jena (2016), a intenção de sair da empresa pode ser descrita como construto de natureza cognitiva que envolve pensamentos relacionados à saída do emprego e busca de outras opções de trabalho. Desse modo, envolve tanto pensamentos de saída como ações de planejamento e vontade de concretizá-los (Siqueira, Gomide Jr., Oliveira \& Polizzi Filho, 2014).

Quando concretizada, a saída dos empregados da organização acarreta, como consequências adicionais, a perda do know how e de parte da história da organização, quebra de processos de trabalho, perda de contatos com clientes e supervisores (Siqueira et al., 2014), além de poder gerar um clima de insegurança entre os empregados que ficam. Essas possíveis derivações destacam a importância de estudar as intenções de rotatividade, defendida como sendo uma das consequências da liderança abusiva, pois está proporcionalmente relacionada com essa.

Buscando verificar o poder explicativo da supervisão abusiva em relação à intenção de deixar a empresa, ou intenção de rotatividade, Harvey, Stoner, Hochwarter e Kacmar (2007) realizaram um estudo empírico cujos resultados mostraram associação positiva, revelando que a percepção de abuso está associada à intenção de deixar a empresa. Adicionalmente, os autores constataram que afetos (positivos) e estratégias pessoais de influência social desempenharam o papel de variáveis moderadoras, de tal sorte que, na presença de abuso, a intenção de saída foi mais forte quando os afetos positivos eram baixos, assim como as estratégias de influência pessoal.

De maneira similar, Pradhan e Jena (2016) focam na relação entre o comportamento abusivo do supervisor e a intenção de rotatividade, e propõem um modelo teórico explicativo que aborda a relação entre essas variáveis tomando como base a teoria de justiça sendo, desse modo, aderente à lógica teórica que sustenta este estudo. Todavia, essa proposição defende a participação de outras variáveis de natureza pessoal e organizacional capazes de mitigar o efeito do abuso percebido e, desse modo, reduzir a probabilidade de que dele derive a saída do trabalhador da empresa. Ainda que se trate de um modelo teórico sem testagem empírica, a lógica subjacente às relações defendidas é similar às associações defendidas nos artigos visitados para a construção deste manuscrito e, dessa forma, constituem contribuições importantes na defesa dos consequentes da liderança abusiva.

Embora a revisão de estudos da área antes referenciados demonstre a crescente atenção que esse fenômeno tem despertado, pesquisadores também não se isentam em questionar as relações defendidas de tal sorte que, por exemplo, indaga-se em que medida a liderança abusiva é antecedente do baixo desempenho, ou ainda, ventila-se a possibilidade de a (baixa) satisfação com o trabalho predizer a percepção de comportamentos abusivos por parte do líder (Martinko, Harvey, Brees \& Mackey, 2013). Alguns desses questionamentos estão acompanhados de achados empíricos que mostram, por exemplo, que o valor instrumental do abuso pode ser questionado, pois é cometido contra trabalhadores com elevado desempenho sendo, nesse caso, desnecessário seu uso como ferramenta que poderia levar à melhora da performance (Walter et al., 2015).

Esse conjunto de achados torna pertinente defender a necessidade de aprofundar no estudo da liderança abusiva, sua natureza, consequentes e antecedentes. Assim, a verificação das evidências de validade da medida, objetivo central deste estudo, constitui um primeiro passo como contribuição ao campo. Ainda, tomando como base os resultados de pesquisas antes descritos, é pertinente defender que a liderança abusiva está associada com outros fenômenos, conforme destacado a seguir:

$\mathrm{H}_{1}$ - Liderança abusiva estará negativamente correlacionada com satisfação no trabalho.

$\mathrm{H}_{2}$ - Liderança abusiva estará positivamente associada com intenção de rotatividade.

\section{Método}

Para a verificação das evidências de validade da medida de liderança abusiva, foi realizada uma pesquisa empírica caracterizada conforme descrito a seguir.

\section{Participantes}

Os dados levantados foram oriundos de um total de 363 respondentes, sendo esses participantes de cursos de capacitação ofertados por instituição de formação de servidores públicos, vinculados, portanto, a diversas organizações. A instituição de formação era localizada na cidade de Brasília, no Distrito Federal. A média de idade dos participantes da pesquisa foi de 39,13 anos $(D P=9,45)$, a maior parte era do sexo masculino, mas a distribuição pode ser descrita como equilibrada (52,6\% masculino). Grande parte da amostra era composta por servidores ou empregados públicos (94,5\%), e a maioria era servidor do poder executivo (86,2\%). Quanto à escolaridade, a formação mais recorrente foi curso de especialização 
(36,1\%), seguido pelo grupo que possuía graduação completa $(16,0 \%)$ ou mestrado completo $(15,4 \%)$. Graus educacionais acima e abaixo dos ora relatados apareceram em porcentagens inferiores a $5 \%$. Com relação à antiguidade na empresa, o valor médio foi 8,3 anos $(D P=8,8)$ e o tempo que trabalham com a mesma chefia teve valor médio de 2,11 anos e desvio padrão de 2,74. Por fim, foi constatado que a maior parte dos participantes estava sob comando de uma pessoa do sexo masculino $(61,2 \%)$.

\section{Procedimentos}

A escala original apresentada por Tepper (2000), composta por 15 afirmativas, foi submetida a procedimento de back-translation. Assim, uma pessoa fluente em línguas inglesa e portuguesa fez a tradução para o português e outra pessoa, também fluente em ambas as línguas, fez a nova tradução ao inglês. As duas versões foram comparadas buscando indagar a sua equivalência. Posteriormente, a versão em língua portuguesa foi revisada por grupo de pesquisadores (de júnior a sênior) visando analisar em que medida a redação capturava o fenômeno, da forma mais clara possível. Nenhum item foi retirado e, desse modo, a escala foi considerada pronta para aplicação. $\mathrm{O}$ acesso aos participantes da pesquisa ocorreu após autorização da instituição de capacitação que oferta os cursos a servidores públicos. Com a anuência obtida, a aplicação foi realizada em sala de aula, utilizando o formato papel e lápis. Prévio à distribuição dos questionários, foram oferecidos esclarecimentos sobre o teor da pesquisa, o seu caráter voluntário, anônimo e sobre o fato dos dados pessoais serem tratados de maneira sigilosa, não permitindo a identificação individualizada dos participantes. Também foi enfatizado que a participação na pesquisa não traria qualquer consequência danosa e que poderiam deixar de participar a qualquer momento. Ao fornecer todas essas informações, os princípios éticos que regem as pesquisas com seres humanos foram respeitadas. As pessoas que voluntariamente concordaram em participar receberam o questionário que, uma vez preenchido, foi devolvido ao pesquisador.

\section{Instrumentos}

Os questionários aplicados eram autoexplicativos e as perguntas de cada escala foram antecedidas de uma breve apresentação das opções de resposta. A escala de liderança abusiva, composta por 15 afirmações, é respondida em escala de frequência de cinco pontos. $\mathrm{O}$ tipo de item constitutivo da medida está exemplificado a seguir: "Meu chefe faz comentários negativos a meu respeito para outras pessoas". A medida original (Tepper, 2000) tem apresentado tanto solução unifatorial como bifatorial, sendo a unifatorial mais frequentemente relatada (Makey et al., 2017). Adicionalmente foi aplicada uma escala de satisfação no trabalho composta por 24 itens distribuídos em cinco fatores sendo esses: satisfação com a chefia, os colegas, as tarefas, as promoções e o salário ( $\alpha$ entre 0,76 e 0,90; Coelho Junior \& Faiad, 2012). As respostas são dadas em escala de sete pontos, do tipo Likert de intensidade (totalmente insatisfeito a totalmente satisfeito). Considerando a adequação das propriedades psicométricas da medida em estudos anteriores, ela foi adotada para investigar associações com a escala de liderança abusiva. Um exemplo dos itens dessa medida indaga: "No meu trabalho atual, sinto-me (resposta) com a maneira como a empresa realiza promoções de seu pessoal". Uma terceira escala aplicada foi a de intenção de rotatividade (Siqueira et al., 2014) composta por três itens que indagam sobre pensamentos, vontades e ações dirigidas à intenção de sair da empresa. O referente original (deixar a empresa) foi alterado de tal sorte a indagar à intenção de sair da equipe. Essa alteração justifica-se pelo fato da amostra estar composta prioritariamente por servidores públicos, que valoram a estabilidade e que, se necessário, tendem a buscar transferências de setores (ou equipes), mais que saída das instituições em que trabalham. O item a seguir exemplifica o tipo de indagação realizada por esta escala: "Tenho vontade de sair da equipe em que trabalho" A escala de respostas dessa medida é de frequência, de cinco pontos e a confiabilidade obtida em estudo realizado pelos autores foi de 0,95 . Por fim, foram levantados dados sociodemográficos e funcionais que permitiram caracterizar a amostra.

\section{Análise de dados}

As estratégias analíticas adotadas foram aquelas que fornecem evidências de validade da medida, na amostra de respondentes desta pesquisa. Assim, foram realizados procedimentos de análise fatorial exploratória (AFE) buscando identificar a melhor organização dos itens em fatores, seguidos da verificação da confiabilidade das soluções fatoriais retidas. A seguir, buscou-se identificar a intensidade da correlação entre a medida de liderança abusiva e as de satisfação e intenção de rotatividade. Para a realização dessas análises, utilizou-se o software SPSS, na versão 23 .

\section{Resultados}

Em primeiro lugar foi investigada a fatorabilidade da medida de liderança abusiva. Os resultados obtidos na análise do $\operatorname{KMO}(0,92)$, do teste de esfericidade $(p<0,01)$ e do determinante da matriz $(2,205 \mathrm{E}-5)$ sugeriram ser pertinente buscar reduzir a massa de dados a fatores. Adicionalmente, foi realizada a inspeção da matriz de correlações, exercício que revelou associações significativas positivas entre todos os itens da escala, com valores que oscilaram entre 0,25 e 0,78 (todos positivos), o que reforça a pertinência de buscar congregar os itens em fatores.

A seguir, buscou-se definir o número adequado de fatores a reter. Para tanto foram considerados critérios 
tanto teóricos como estatísticos. A adoção do critério de Guttman-Kaiser, pautado na magnitude do eigenvalue (valor próprio) demonstra que a matriz de dados tolera até o máximo de dois fatores. A este respeito ainda é necessário destacar que Laros (2012) alerta que o uso desse critério, como indicador do número adequado de fatores a reter, resulta em superextração (portanto, número errado) de fatores, em $78 \%$ das vezes em que é aplicado. Outro critério utilizado foi o gráfico do scree, que sugere a pertinência de reter apenas um fator. Quanto ao critério teórico, a revisão das pesquisas realizadas sobre a temática, revela que boa parte delas adota uma solução unifatorial, fato que reflete a estrutura unidimensional do construto (Mackey et al., 2017), conforme proposta original realizada por Tepper (2000). Desse modo, considerando o conjunto de evidências obtidas, optou-se por uma solução unifatorial da medida. Ainda assim, investigou-se a estrutura fatorial organizada em dois fatores. Essa solução mostrou-se inadequada, uma vez que os fatores resultantes foram teoricamente não interpretáveis e surgiram itens complexos com cargas fatoriais, em ambos os fatores, superiores a 0,3 . Portanto, a solução bifatorial foi descartada em favor da unifatorial. A Tabela 1 mostra os resultados da solução mais satisfatória, para a amostra em que foi testada.

Tabela 1

Solução Fatorial da Medida de Liderança Abusiva

\begin{tabular}{|c|c|}
\hline $\begin{array}{c}\text { Descrição do enunciado } \\
\text { Meu chefe... }\end{array}$ & $\begin{array}{c}\text { Carga } \\
\text { Fatorial }\end{array}$ \\
\hline 4... me diminui na presença de outras pessoas. & 0,86 \\
\hline 1... me ridiculariza. & 0,82 \\
\hline 11... faz comentários negativos a meu respeito para outras pessoas. & 0,81 \\
\hline 2... me diz que meus pensamentos ou sentimentos são irracionais. & 0,78 \\
\hline 12... é rude comigo. & 0,78 \\
\hline 8... me culpa para evitar se constranger. & 0,76 \\
\hline 10... "desconta" sua raiva em mim quando está irritado por outro motivo. & 0,75 \\
\hline 15... mente pra mim. & 0,74 \\
\hline 5... invade minha privacidade. & 0,73 \\
\hline $6 \ldots$ me lembra de erros e falhas que cometi no passado. & 0,70 \\
\hline 9... não cumpre acordos que ele(a) faz comigo. & 0,69 \\
\hline $14 \ldots$ me diz que sou incompetente. & 0,68 \\
\hline 13... impede que eu interaja com meus colegas de trabalho. & 0,67 \\
\hline 3... me trata de maneira fria. & 0,66 \\
\hline 7... não me dá crédito por trabalhos que realizei que exigiram grande esforço. & 0,52 \\
\hline $\mathrm{N}^{\circ}$ de itens & 15 \\
\hline Alfa de Cronbach $(\alpha)$ & 0,94 \\
\hline Média da correlação item-total & 0,70 \\
\hline
\end{tabular}

Conforme a Tabela 1 mostra, as cargas fatoriais superam longamente os critérios apontados como aceitáveis (Tabachncik \& Fidel, 2007) para serem consideradas contribuições minimamente relevantes $(0,30)$ na avaliação do construto em foco. Os resultados obtidos demonstram que os itens que melhor capturam os comportamentos abusivos dos líderes, conforme percebidos pelos trabalhadores, são aqueles que descrevem abuso cometido em público ou perante outras pessoas ("me diminui perante outras pessoas", ou ainda, "faz comentários negativos perante outras pessoas”).

A opção de reter um único fator resultou na obtenção de estrutura que captura $56,79 \%$ da variância do fenômeno, sem perda de qualquer item. Tendo optado por essa solução, foi investigada, a confiabilidade do fator. Para tanto, foi calculado o valor do alfa de Cronbach e a magnitude do valor médio da correlação item total (corrigida). Em ambos os casos, os resultados foram satisfatórios $\left(\alpha=0,94 ; M_{\text {item-total }}=0,70\right.$-corrigida- $)$ e evidenciaram a pertinência da solução adotada.

Uma vez tendo identificado a adequabilidade da estrutura unifatorial da medida de liderança abusiva foi investigada a sua correlação com outros construtos teoricamente vinculados, de sorte a testar a pertinência das hipóteses de associação levantadas. Para tanto, foi realizado teste de correlação de Pearson entre liderança abusiva, de um lado, e satisfação e intenção de rotatividade, do outro.

Incialmente, entretanto, foi verificada a adequação da media de satisfação para a amostra deste estudo. A 
satisfação com o trabalho foi avaliada mediante o uso de uma escala de estrutura multidimensional cuja análise, na amostra de participantes da presente pesquisa, evidenciou a presença de cinco fatores: salário (F1; $\alpha=0,93 ; M_{\text {item- }}$ ${ }_{\text {total }}=0,83$-corrigida- $)$, chefia $\left(\mathrm{F} 2 ; \alpha=0,93 ; M_{\text {item-total }}=0,82\right.$ -corrigida-), promoções (F3; $\alpha=0,92 ; M_{\text {item-total }}=0,79$ -corrigida-), colegas (F4; $\alpha=0,88 ; M_{\text {item-total }}=0,73$-corrigida-) e tarefas (F5; $\alpha=0,86 ; M_{\text {item-total }}=0,71$-corrigida-). Portanto, foram similares aos vistos em estudos realizados usando a mesma medida (Coelho Junior \& Faiad, 2012).

$\mathrm{Na}$ testagem da associação defendida na primeira hipótese $\left(\mathrm{H}_{1}\right)$, que apontava correlação negativa entre percepção de abuso e satisfação, os resultados demonstraram que a relação mais forte foi com o fator satisfação com a chefia $(r=-0,58)$, e as menos intensas ocorreram com os fatores satisfação com os colegas $(r=-0,15)$, com as tarefas $(r=-0,18)$ e com as promoções $(r=-0,18)$. Com o fator salário, a associação foi ausente $(p>0,1)$. Esse conjunto de dados demonstra, portanto, que as pessoas que se percebem como alvos de comportamentos abusivos, por parte dos seus chefes imediatos, tendem a relatar também que estão menos satisfeitos com as chefias, os colegas, as tarefas e as políticas de promoção da empresa e permitem corroborar a $\mathrm{H}_{1}$.

A segunda hipótese $\left(\mathrm{H}_{2}\right)$ defendeu associação positiva entre liderança abusiva e intenção de rotatividade. A rotatividade foi avaliada por medida unidimensional que mostrou índices adequados de confiabilidade na amostra deste estudo $\left(\alpha=0,92 ; M_{\text {item-total }}=0,83\right.$-corrigida-). A testagem da hipótese em tela foi realizada mediante o cálculo da correlação de Pearson que apresentou valores significativos positivos $(r=0,27)$. Desse modo, foi constatado que os respondentes que perceberam comportamentos abusivos dos seus superiores declararam, também. O conjunto de associações identificadas é o mostrado na Tabela 2.

Tabela 2

Média Aritmética, Desvios e Correlações entre as Variáveis do Estudo

\begin{tabular}{|c|c|c|c|c|c|c|c|c|}
\hline & $M$ & $D P$ & 1 & 2 & 3 & 4 & 5 & 6 \\
\hline L. Abusiva & 1,40 & 0,61 & - & & & & & \\
\hline Sat F1 & 4,28 & 1,74 & $-0,09$ & - & & & & \\
\hline Sat F2 & 4,94 & 1,61 & $-0,58^{*}$ & $0,24^{*}$ & - & & & \\
\hline Sat F3 & 3,47 & 1,69 & $-0,18^{*}$ & $0,57^{*}$ & $0,42^{*}$ & - & & \\
\hline Sat F4 & 5,30 & 1,26 & $-0,15^{*}$ & $0,31^{*}$ & $0,49^{*}$ & $0,38^{*}$ & - & \\
\hline Sat F5 & 4,81 & 1,46 & $-0,18^{*}$ & $0,31^{*}$ & $0,49^{*}$ & $0,53^{*}$ & $0,49^{*}$ & - \\
\hline Int. Rota. & 2,90 & 1,29 & $0,27^{*}$ & $-0,17^{*}$ & $-0,48$ & $-0,33^{*}$ & $-0,27^{*}$ & $-0,56^{*}$ \\
\hline
\end{tabular}

Nota. ${ }^{*} p<0,01$; Satisfação: F1=Salário; F2=Chefia; F3=Promoções; F4=Colegas; F5=Tarefas

Os dados da Tabela 2 também evidenciam que os respondentes declaram estar, no geral, razoavelmente satisfeitos com os diversos aspectos do seu trabalho, pois o maior valor é de 5,30 (escala de respostas de sete pontos), ter intenções médias de rotatividade (2,90; escala de respostas de 5 pontos) e, no geral, terem sofrido escasso abuso por parte dos lideres $(1,40)$. Esse conjunto de dados, relativos tanto às características da medida de liderança abusiva, como as correlações encontradas com a satisfação e a intenção de rotatividade eliciam teorizações quanto ao comportamento da escala objeto deste estudo. Assim, a próxima seção discute esse conjunto de achados à luz de teorias do campo organizacional.

\section{Discussão}

A ocorrência de comportamentos abusivos, por parte dos líderes, constitui um desvio daquilo que as teorias de liderança defendem como atuação esperada desses atores. Embora a função do líder seja alavancar a efetividade da organização, eliciando contribuições individuais e coletivas, não se espera que isso ocorra a qualquer custo ou mediante a manifestação de comportamentos descritos como abusivos. Ainda assim, não parece razoável afirmar que a liderança abusiva não esteja presente no cenário organizacional. Portanto, é de fundamental importância compreender a ocorrência desse fenômeno, entender a sua natureza e, a partir disso, investigar tanto seus antecedentes como os impactos que dele derivam.

Ao discutir a natureza do construto, Tepper (2000, 2007) destaca que sua manifestação está pautada na percepção de comportamentos como ridicularizar o subordinado, fazer dele um bode expiatório, não reconhecer as contribuições feitas à organização, humilhação e intimidação publicamente manifestada, conforme defendido também por outros autores da área (Pradhan \& Jena, 2016).

A escala proposta por Tepper (2000) condensa um total de 15 afirmações. Pesquisas da área têm utilizado tanto a medida completa (Khan, et al., 2016; Zhang et al., 2014) como versões reduzidas (Kernan et al., 2011). Na pesquisa objeto deste relato, todos os itens foram mantidos. A pertinência dessa decisão encontra sustento nos 
resultados obtidos, pois, conforme mostra a Tabela 1, a menor carga fatorial foi de 0,52. Esse dado evidencia que todos os itens contribuem à mensuração do construto latente. Conforme Pasquali (2010), os itens constitutivos de uma medida devem cobrir um espectro específico no campo teórico do fenômeno focado. Os resultados obtidos na análise fatorial exploratória mostram que cada afirmação ou item da escala é relevante, devendo, portanto, ser retida. Isso porque todos superam o valor de carga fatorial apontado como mínimo $(0,30)$ para que um item possa ser considerado relevante para a mensuração de um construto (Tabachnick \& Fidel, 2007). A pertinência da decisão também está corroborada pela análise de confiabilidade realizada, uma vez que não foram encontradas evidências empíricas sobre possíveis benefícios resultantes da retirada de qualquer item, seja em termos de acréscimo do valor do alfa de Cronbach ou da melhora da magnitude média da correlação item total corrigida. O valor do alfa, nesta pesquisa $(0,94)$, mostrou-se similar ao reportado em outras pesquisas da área (Makey et al., 2017), dado esse que permite concluir que o processo de tradução das afirmativas que compõem a medida foi adequado, de tal sorte que o conteúdo abordado pela escala original foi preservado, permitindo, desse modo, capturar o construto latente. Além dos resultados favoráveis da solução unifatorial, os pobres resultados da solução bifatorial reforçam a pertinência de condensar os 15 itens em um fator único. É importante destacar que Tabachnick e Fidel (2007) enfatizam que os fatores extraídos precisam ser teoricamente interpretáveis, além de psicometricamente adequados, para que se conclua sobre a pertinência da solução da medida. Estes resultados eliciam reflexões sobre as proposições de solução bifatorial encontradas na literatura (Makey et al., 2017), pois essa se mostrou improcedente na amostra de respondentes com que este estudo foi realizado. Assim, indaga-se quanto à presença de efeitos oriundos de variáveis não estudadas, como as relativas à cultura que podem ter favorecido a condensação dos 15 itens em um conjunto único.

Focando, agora, nos valores alcançados nas avaliações dos diferentes construtos investigados, chama a atenção o baixo valor da média aritmética da liderança abusiva, que é de apenas $1,40(D P=0,61)$ em uma escala de 5 pontos. A esse respeito, cabe apontar que ele não é diferente dos valores médios reportados em metanálise que envolveu amostras de diferentes países (Mackey et al., 2017). Nesse estudo, ao comparar valores médios alcançados em amostras de diferentes nações foram encontradas médias aritméticas que oscilaram entre 2,17 e 1,32 . Assim, o valor médio da amostra brasileira mostra-se semelhante ao visto em pesquisas de natureza similar, realizados em diferentes países do mundo. Esses achados demonstram que, no geral, há baixa prevalência de comportamentos (ou percepção) de abuso por parte das lideranças organizacionais. Ainda assim, é importante atentar para os resultados nocivos que deles derivam.
Analisando o conteúdo abordado pelas afirmações que compõem a escala observa-se que aquelas com cargas mais elevadas, portanto, as que melhor representam o construto, fazem referência a abusos cometidos perante outras pessoas. Isso é aderente à lógica teórica da liderança abusiva, que possui como um dos seus atributos centrais o caráter funcional. Ou seja, essa manifestação de abuso teria como função exemplificar perante outros um comportamento considerado indesejado (Tepper, 2000).

Outra característica do abuso é o fato de ser percebido pelo trabalhador. Esse aspecto é observado analisando o enunciado dos itens e a escala de resposta associada. As assertivas da medida indagam o respondente sobre a frequência com que o chefe demonstra os comportamentos descritos; desse modo, o caráter subjetivo é capturado. Pesquisas da área mencionam a centralidade desse aspecto na compreensão das consequências da liderança abusiva, pois é imprescindível ter em mente que não é o comportamento abusivo do líder o que desencadeia consequências no nível organizacional e individual, mas a percepção do trabalhador sobre a ocorrência do abuso (Martinko et al., 2013). Com relação às opções de resposta, ao estarem em formato de frequência, permitem abordar também a continuidade do abuso que constitui atributo identitário do fenômeno sob análise.

Por fim, o conjunto de descrições de possíveis comportamentos de abuso, que constituem os itens da escala de liderança abusiva, não abordam situações de contato físico. Desse modo, a quarta característica do construto é respeitada pela medida. Quanto a ela, Tepper (2000, 2007) enfatiza que abuso físico representa bullying ou agressão no trabalho.

A discussão das propriedades teóricas da liderança abusiva, associada à análise do conteúdo e comportamento dos itens presentes na medida, permite afirmar que os resultados obtidos oferecem indícios consistentes de validade de conteúdo da escala. Portanto, considera-se que se trata de instrumento adequado para capturar o fenômeno descrito como liderança abusiva ocorrida no cenário organizacional.

Além de investigar o comportamento dos itens e a sua pertinência para avaliar o fenômeno em tela, foram analisadas as associações com outros construtos presentes no cenário organizacional. Conforme mostram os resultados da Tabela 2, a liderança abusiva esteve associada à satisfação. Entretanto, tendo em vista a natureza multidimensional da medida, é importante observar as associações diferenciadas mantidas com cada fator. Assim, a correlação mais forte se deu como segundo fator (F2: satisfação com a chefia). Esse fato destaca novamente a pertinência da medida uma vez que, tanto a liderança abusiva, como o fator dois da satisfação, possuem o mesmo referente: o chefe. Os dados também mostram uma associação ausente e merece ser discutida. Trata-se da correlação com F1, relativo à satisfação com o salário. 
Isso significa que o fato de perceber comportamentos abusivos do líder não está associado à satisfação reportada com o salário. Essa ausência de vinculação pode ser explicada pelo perfil da amostra de respondentes, pois quase a totalidade desses são servidores e empregados públicos $(94,5 \%)$, portanto, os seus salários dependem do poder (executivo, legislativo ou judiciário) ao que se vinculam, além do plano da sua carreira.

Discutindo as bases teóricas que justificam a associação entre liderança abusiva e satisfação, Kernan et al. (2011) recorrem à justiça interacional e destacam que princípios gerais sobre a forma justa de ser tratado (expectativas) são violadas quando o líder, na condição de representante da organização, demonstra comportamentos contrários aos esperados. Quando essa vivência (percebida) se prolonga, é de se esperar que sentimentos de frustração surjam na medida em que o observado não corresponde ao esperado. Assim, a insatisfação seria uma consequência natural, especialmente se consideramos que o foco da satisfação é o chefe (F2). Essa associação relatada pode até desencadear a dúvida, por parte do leitor, quanto ao fato da liderança abusiva e a falta de satisfação com a chefia evidenciarem os lados opostos de uma mesma moeda. Essa dúvida pode ser dirimida ao observar tanto o conteúdo dos itens das escalas, como a magnitude da correlação $(r=-0,56)$. Conforme já manifestado, as assertivas que compõem o instrumento de liderança abusiva descrevem comportamentos do supervisor, na relação mantida com o subordinado. A medida de satisfação, por sua vez, indaga sobre o comportamento geral do líder, focando também nas suas competências gerenciais. Com relação à magnitude do efeito, o valor encontrado revela que liderança abusiva e satisfação com o chefe compartilham 31,3\% da variância. Assim, ainda existe ao redor de $70 \%$ de conteúdo que é específico de cada construto. O valor antes mencionado é superior ao reportado em estudos da área ( $r=-0,31$; Mackey et al., 2017) contudo, cabe destacar o fato desses abordarem satisfação no trabalho, de maneira geral, e não com a chefia como feito neste estudo. Assim, é pertinente concluir que a associação identificada, a qual forneceu suporte para a $\mathrm{H}_{1}$, era a esperada para esses construtos.

Neste estudo também foi investigada a correlação com a intenção de saída da equipe (intenção de rotatividade). Cabe lembrar que a intenção de saída foi descrita como a vontade do trabalhador de deixar a equipe, a qual pode ou não se concretizar, a depender da participação de outros fatores intervenientes como a natureza do trabalho e a qualificação do trabalhador. Ainda que não se concretize a saída, a vontade existe e ela pode incrementar na medida em que as condições de trabalho não atendam às expectativas do trabalhador.

$\mathrm{Na}$ ocorrência de comportamentos de abuso por parte do líder, é de se esperar que o trabalhador busque arranjos que diminuam a probabilidade de ser vítima do abuso. Isso pode ser traduzido no surgimento da vontade de mudar de equipe de trabalho. A defesa dessa associação já foi trazida em estudos teóricos (Pradhan \& Jena, 2016) e empíricos (Tepper, Carr, Breaux, Geider, Hu \& Hua, 2009), embora esses enfatizem a intenção de sair da empresa. De qualquer forma, a revisão dos achados dessas pesquisas demonstra que relações significativas foram identificadas entre percepção de comportamentos abusivos e intenção de rotatividade (Rodwell, Brunetto, Demir, Shacklock \& Farr-Wharton, 2014).

Nos resultados desta pesquisa foi observada relação direta entre liderança abusiva e intenção de sair da equipe, achado esse que deu suporte à $\mathrm{H}_{2}$. A magnitude do efeito $(p<0,01)$ foi de 0,28 e demonstra que a percepção do trabalhador de ser vítima de abuso, por parte do seu chefe imediato, está associada a aproximadamente 9\% da intenção do empregado de deixar a equipe.

Os achados relacionados à associação entre a liderança abusiva e outros construtos - a satisfação e a intenção de deixar a equipe - oferecem indícios adicionais da validade da medida traduzida (Tepper, 2000), uma vez que foram constatadas as relações esperadas (H1 e H2) tendo em vista a natureza dos comportamentos de abuso percebidos pelo trabalhador.

Desde uma perspectiva prática, a disponibilização de um instrumento que permite diagnosticar a ocorrência de liderança abusiva, no ambiente organizacional, é certamente uma contribuição relevante ao campo. Isso porque, ainda que a atuação esperada do líder seja apoiar os trabalhadores e a própria empresa no alcance de objetivos comuns (profissionais e organizacionais), é fato que o abuso ocorre e não seria apropriado negar existência desse problema. Todavia, conforme apontado neste manuscrito, o foco de análise não é o comportamento abusivo em si, mas a percepção do comportamento do líder como sendo abusivo. Assim, é imprescindível ter acesso aos trabalhadores para que possam relatar as suas experiências com os seus superiores imediatos. Contudo, dada a sensibilidade do assunto, existem diversas dificuldades associadas à discussão aberta dessas situações. Por essa razão, é de extrema importância contar com uma medida que permita, via questionário padronizado, levantar as percepções diretamente dos trabalhadores sobre a ocorrência do abuso, fato que constitui uma contribuição prática para os gestores, derivada desta pesquisa.

$\mathrm{E}$, quanto às ações que podem derivar do diagnóstico da ocorrência do abuso, elas devem ser desenhadas de acordo com as características, em termos de profundidade e abrangência, do problema identificado. Para tanto, faz-se imprescindível a identificação de graus diferenciados de abuso, em setores diferentes da organização ou por grupos de trabalhadores. Essa identificação é viabilizada mediante o levantamento de dados sociodemográficos e funcionais, pois esses permitem análises pormenorizadas por grupos, os que podem ou não, perceber de maneira diferenciada a ocorrência do abuso. 
Embora este estudo traga contribuições práticas, ele não está isento de limitações. Uma delas é a especificidade da amostra que, ao estar composta quase em sua integralidade por servidores públicos, permite questionar se o comportamento da medida irá se replicar ao ser utilizada em outros contextos organizacionais ou empresariais. Ainda assim, o fato dos resultados terem sido similares aos observados em outros estudos (Mackey et al., 2017) oferece indícios sobre a adequação da medida para o fim a que se destina.

Com relação às associações identificadas entre a medida de liderança abusiva e construtos como satisfação e intenção de rotatividade, a lógica teórica subjacente é a defesa da atuação do abuso percebido como um fato desencadeante dos outros dois. Contudo, as estratégias analíticas utilizadas (correlações) mostram apenas associação entre os fenômenos, não sendo possível estabelecer relações de causalidade entre eles. Por tanto, essas associações precisam ser mais bem investigadas em estudos futuros, ainda que elas tenham sido longamente defendidas e evidenciadas em pesquisas tanto teórica como empírica (Tepper, 2000, 2007, Kernan et al., 2011, Martinko et al., 2013).

Pontualmente, focando nos próximos passos no estudo da ocorrência da liderança abusiva, é importante destacar a necessidade de que mais estudos sejam realizados no Brasil, buscando identificar a estabilidade da medida original de Tepper (2000). Adicionalmente, ainda quanto à medida, é necessário investigar se a solução de dois fatores, de fato, não emerge quando aplicada em amostras de trabalhadores com outro perfil sociodemográfico. Com relação aos efeitos da ocorrência de liderança abusiva, é importante que estudos futuros considerem a intervenção de outras variáveis, como as culturais, pois padrões específicos de comportamento podem estar atrelados a valores dos brasileiros, e esses podem afetar a intensidade e a natureza das relações entre liderança abusiva e seus consequentes. Por fim, estudos de desenho longitudinal podem ajudar a esclarecer as dúvidas ainda presentes quanto à definição de antecedentes e consequentes, nos estudos realizados sobre a liderança abusiva.

Ainda com essas limitações, considera-se que a temática é relevante, tendo em vista a severidade das possíveis consequências tanto para o trabalhador como para a organização. Assim, espera-se que este estudo contribua para abrir um novo caminho na busca da melhor compreensão de comportamentos desviantes ocorridos nas organizações.

\section{Referências}

Bies, R. J. (2000). Interactional (in)justice: The sacred and the profane. Em J. Greenberg, \& R. Cropanzano (Eds.), Advances in organizational behavior: Forthcoming. Stanford, CA: Stanford University Press.

Borges, E. \& Ferreira, T. (2015). Bullying no trabalho: Adaptação do Negative Acts Questionnaire-Revised (NAQ-R) em enfermeiros. Revista Portuguesa de Enfermagem de Saúde Mental, (13), 25-33. Recuperado de http://www.scielo.mec.pt/scielo.php?script=sci_ abstract\&pid $=$ S1647-21602015000200004\&lng $=$ pt\&nrm $=\mathrm{i}$

Borges, L. O., Guimaraes, L. A. M., \& Souza, S. (2013). Diagnóstico e promoção da saúde psíquica no trabalho. Em L. Borges, \& L. Mourão (Eds.), O trabalho e as organizações. Porto Alegre: Artmed.

Brasileiros são os mais estressados do mundo nos ambientes de trabalho. (2016, Novembro). SaudeOcupacional.org. Recuperado de https:// www.saudeocupacional.org/2016/11/brasileiros-sao-os-mais-estressados-do-mundo-nos-ambientes-de-trabalho.html

Burton, J., Hoobler, J., \& Scheuer, M. (2012). Supervisor workplace stress and abusive supervision: The buffering effect of exercise. Journal of Business and Psychology, 27(3), 271-279. doi:10.1007/s10869-011-9255-0

Coelho Junior, F., \& Faiad, C. (2012). Evidências de validade da escala de satisfação no trabalho. Avaliação Psicológica, (11), 111-121. Recuperado de http://pepsic.bvsalud.org/scielo.php?script=sci_arttext\&pid=S1677-04712012000100011

Einarsen, S., Hoel, H., Zapf, D., \& Cooper, C. (2011). Bullying and Harassment in the workplace. Developments in theory, research and practice. New York: Taylor \& Francis. doi:10.1201/EBK1439804896

Garrido, J. C. (2012). Liderança abusiva e consequências negativas para o seguidor/liderado: Um estudo de caso (Dissertação de mestrado). Universidade do Minho. Portugal.

Harvey, P., Stoner, J., Hochwarter, W., \& Kacmar, C. (2007). Coping with abusive supervision: The neutralizing effects of ingratiation and positive affect on negative employee outcomes. The Leadership Quarterly, (18), 264-280. doi:10.1016/j.leaqua.2007.03.008

Hon, A., \& Lu, L. (2016). When will the trickle-down effect of abusive supervision be alleviated? The moderating roles of power distance and traditional cultures. Cornell Hospitality Quarterly, 57(4), 421-433. doi:10.1177/1938965515624013

Kernan, M., Watson, S., Chen, F., \& Kim, T. (2011). How cultural values affect the impact of abusive supervision on worker attitudes. Cross Cultural Management, 18(4), 464-484. doi:10.1108/13527601111179528

Khan, A., Moss, S., Quratulain, S., \& Hameed, I. (2016). When and how subordinate performance leads to abusive supervision: A social dominance perspective. Journal of Management (First published date: June-21), 1-26. doi:10.1177/0149206316653930

Kim, S., Kim, M., \& Yun, S. (2015). Knowledge sharing, abusive supervision, and support: A social exchange perspective. Group E Organization Management, 40(5), 599-624. doi:10.1177/1059601115577514

Laros, J. A. (2012). O uso da análise fatorial: Algumas diretrizes para pesquisadores. Em L. Pasquali (Ed.). Análise Fatorial para pesquisadores. 1ed. Brasília: LabPAM Editora. 141-160.

Mackey, J., Frieder, R., Brees, J., \& Martinko, M. (2017). Abusive supervision: A meta-analysis and empirical review. Journal of Management, 43(6), 1940-1965. doi:10.1177/0149206315573997 
Martinko, M., Sikora, D., \& Harvey, P. (2012). The relationships between attribution styles, LMX, and perceptions of abusive supervision. Journal of Leadership \& Organizational Studies, 19(4), 397-406. doi:10.1177/1548051811435791

Martinko, M., Harvey, P., Brees, J., \& Mackey, J. (2013). A review of abusive supervision research. Journal of Organizational Behavior, (34), S120-S137. doi:10.1002/job.1888

Moreno, J., Herrera, D., \& Gargurevich, R. (2019). Propiedades psicométricas de la versión en español de la escala de supervisión abusiva en población laboral ecuatoriana. Revista Iberoamericana de Diagnóstico y Evaluación, 51(2), 117-133. doi:10.21865/RIDEP51.2.09

Pasquali, L. (2010). Instrumentação Psicológica. Porto Alegre: Artmed.

Pradhan, S., \& Jena, L. (2016). The moderating role of neutralizers on the relationship between abusive supervision and intention to quit: A proposed model. Journal of Human Values, 22(3), 238-248. doi:10.1177/0971685816650575

Rodwell, J., Brunetto, Y., Demir, D., Shacklock, K., \& Farr-Wharton, R. (2014). Abusive supervision and links to nurse intentions to quit. Journal of Nursing Scholarship, (46), 357-365. doi:10.1111/jnu.12089

Siqueira, M. M. Gomide Jr., S., Oliveira, A., \& Polizzi Filho, A. (2014). Intenção de rotatividade. Em M. M. Siqueira (Eds.), Novas medidas de comportamento organizacional (209-216). Porto Alegre: Artmed.

Tabachnick, B. G., \& Fidell, L. S. (2007). Using multivariate statistics (5th ed.). Boston, MA: Allyn \& Bacon.

Tepper, B. J. (2000). Consequences of abusive supervision. Academy of Management Journal, 43(2), 178-190. doi:10.2307/1556375

Tepper, B. J. (2007). Abusive supervision in work organizations: Review, synthesis, and research agenda. Journal of Management, (33), 261289. doi:10.1177/0149206307300812

Tepper, B. J., Carr, J., Breaux, D. M, Geider, S. Hu, C., \& Hua, W. (2009). Abusive supervision, intentions to quit, and employees' workplace deviance: A power/dependence analysis. Organizational Behavior and Human Decision Processes, (109), 156-167. doi:10.1016/j. obhdp.2009.03.004

Walter, F., van der Vegt, G., Huang, X., \& Miao, Q. (2015). Abusive supervision and subordinate performance: Instrumentality considerations in the emergence and consequences of abusive supervision. Journal of Applied Psychology, 100(4), 1056-1072. doi:10.1037/a0038513

Zanelli, J. C. (2015). Estresse nas Organizações de Trabalho. Em Pedro F. Bendassolli \& Jairo Eduardo-Andrade. (Eds.). Dicionário de Psicologia do Trabalho e das Organizações. 1ed. São Paulo: Casapsi Livraria e Editora Ltda. p. 333-339.

Zhang, H., Kwanm, H., Zhang, X., \& Wu, L. (2014). High core self-evaluators maintain creativity: A motivational model of abusive supervision. Journal of Management, 40(4), 1151-1174. doi:10.1177/0149206312460681

Zhaoquan, J., Kwan, H., Qiu, Q., Liu, Z., \& Yim, F. (2012). Abusive supervision and frontline employees' service performance. The Service Industries Journal, 32(5), 683-698. doi:10.1080/02642069.2011.614338

\section{Sobre as autoras}

Katia Elizabeth Puente-Palacios é mestre e doutora em Psicologia pela Universidade de Brasília e pós-doutorado pela Universidade de Valencia-Espanha. Atualmente é professora do Departamento de Psicologia Social e do Trabalho (PST) e do Programa de pós-graduação em Psicologia Social, do Trabalho e das Organizações (PSTO) da Universidade de Brasília.

Marcela Guimarães Côrtes é pesquisadora-tecnologista em Informações e Avaliações Educacionais no INEP. Atualmente é doutoranda em Psicologia Social, do Trabalho e das Organizações pela Universidade de Brasília, com graduação em Fonoaudiologia e mestrado em Ciências da Saúde pela Universidade Federal de Minas Gerais. 